\title{
Integrating media asset collections and production workflow
}

\author{
Kevin Comerford \\ is the Senior Media Archive Manager at Microsoft Studios, where he has directed daily operations of the digital asset \\ management initiative there since 1996. In 1999, he designed and managed the implementation of MSMedia, the \\ corporation's video catalog and order management system. A librarian and artist by training, he was previously the \\ Visual Resources Manager at the Dallas Museum of Art, and served as a database consultant to the Sixth Floor \\ Museum.
}

Keywords: cataloging and indexing systems, DAM, digital video and audio, metadata, video recordings, production and direction, management, workflow systems, case studies

Abstract The video production industry is currently working through an intermediate period between videotape-based and digital production methodologies. This confluence of old and new technologies presents numerous challenges both in the management and retrieval of media content assets. This paper summarizes theoretical issues and examines how the Media Content Management Group at Microsoft Studios has integrated the timely and accurate collection of metadata into the larger video production workflow.

\section{INTRODUCTION}

The video production industry is currently working through an intermediate period between videotapebased and file-based digital production methodologies. While the transition toward file-based production is fully underway, having impacted all production facilities to some extent, the present scale of capital outlay required, as well as the still uneven technological landscape, ensure that the complete transition from old to "new post" will not occur overnight. Thus for the majority of production facilities, conventional tape-based production shows little sign of losing its raison d'être entirely in the foreseeable future. As such, the challenges to practitioners of digital asset management (DAM) and media asset management are not only to ensure the integral restorability and reusability of both tape- and file-based production content, but also to develop integrated strategies for management and access.

MS Studios is an excellent example of an intermediate period production facility. This internal resource supports the presentation, training, marketing and some of the specialized mediaspecific product development functions for Microsoft Corporation, its subsidiaries and partners worldwide. Featuring three sound stages and stateof-the-art video production facilities, MS Studios produces, on average, 200 corporate communications projects each month. The variety of content types and distribution requirements necessitate an equally diverse assortment of production workflows and content- 
handling strategies. Projects which utilize both digital and tape-based source material are not uncommon, and depending on project requirements these elements may be processed sequentially (tape-to-digital), in parallel or asynchronously. Interestingly, the broad distribution requirements for this content frequently require the format cycle to repeat itself (tape-to-digital-totape).

The confluence of digital and taped media elements at MS Studios has likewise been a daily fact of life for its media content management team since the facility was launched over ten years ago. In 1995, a videotape vault facility was established to house interactive television elements produced for one of Microsoft's research initiatives. Then in late 1996, DAM made its debut, originally to quell the flood of still graphics produced for Microsoft's line of consumer CD products (eg Encarta and the Microsoft Home series). Since that time, the media requirements of Microsoft's products, communications and marketing initiatives have evolved and expanded with great alacrity, as have the holdings of MS Studios archives. At present, the videotape archive houses 120,000 production grade videotapes, while the DAM repository has grown to $20 \mathrm{~Tb}$ (over 4 million files) of digital media content. With an average monthly acquisition rate of 1,000 videotapes and over 160,000 individual files, the seven-member media content management team is tasked with the documentation, storage and distribution of a broad array of content resources. Successful management of the constant flow of new acquisitions is dependent upon the timely and accurate collection of asset metadata, and the correlation of related digital and videotape assets.

\section{MEDIA ASSETS AND METADATA}

A rather important characteristic of digital video is that a large percentage of it exists wholly or partially as the product of conversion. At MS Studios, for example, the majority of digital video assets under management were originally encoded from tape.

Significant here is that the digital video assets are homological extensions of their videotape counterparts - the former being an outgrowth of the latter. Given that the relationship between taped and digital video is still somewhat intertwined, it is not surprising that production facilities who employ DAM for video storage also continue to maintain active production videotape archives. Consequently, in production environments where neither taped nor digital video currently maintain functional precedence over the other, the larger collections of these assets will continue to grow in tandem and require separate resource commitments to maintain. While this is not a problem per se, it is important to avoid the unfortunate tendency towards perceiving each collection as an isolated entity, the management of which is an adjunct issue. Rather, until an actual date can be set after which videotape becomes officially obsolete, both taped and digital video assets should be administered as an integrated resource. Particularly in terms of content access and metadata management, this is why a comprehensive asset management strategy is necessary to ensure that all resources are properly correlated.

The term correlation should be clarified 
here to imply a unified approach to the accumulated metadata of both the videotape and digital asset collections. In conventional terms, each collection is administered through the auspices of some form of catalog. The concept of the catalog is universal to information management disciplines, including DAM. To use a lofty metaphor, the catalog is akin to the figure of Atlas supporting the world on his shoulders. Because information objects (eg books, videotapes, computer files) are opaque by nature and analog in presentation format, when a collection of information objects is absent, a representational index (in effect, without a surrogation of the original content), those objects are just as lost to use as the world would be lost without Atlas to suspend it over the abyss. Before digressing too far upon the image of the mythological entity who suffers an acute allergy to book dust, it is germane to note that while the catalog is the foundation of any collection of information objects, the underpinnings of the catalog are of course standards of structure and practice. A catalog cannot function without standards that control the configuration of records, which govern the rules and practice of data entry, that shape and define the vocabulary which provides an ontological framework for the content described. Standards are the fulcrum point between successful and unsuccessful information retrieval, between the wasteland of precision and the inundation of recall.

While the nomenclature of DAM differs to an extent from that of established library science, the fundamental conventions used to manage corporeal information objects are more similar to those applied to digital assets than they are different. In fact, these descriptive conventions are concomitant entities, those which can be applied to DAM systems being shadowy reflections of their ancestral counterparts. Whereas in a library catalog one consults a bibliographic record, in a DAM system one consults a metadata record; both entities provide access to the information object being described, albeit via a surrogate. The one radical difference extant in DAM technology is that for all practical purposes the catalog and the collection are integrated and essentially synonymous. By contrast a library or archive catalog is conspicuously separated and abstracted from the collection it describes. As such, the DAM catalog model, by and large, offers a distinct advantage over its bibliographic country cousin. However, as many conventional library catalog systems now provide resource linking and previewing capabilities, it seems appropriate to invent a new designation to call out the unique architectural differences of the DAM catalog paradigm - dare I suggest metadex?

\section{METADATA AND DAM}

With regard to the creation and maintenance of catalog and metadata records, there are significant differences between these descriptive methodologies which are important to note. Standards that govern library catalogs and cataloging practices are decidedly institutionalized, highly regulated, and more apt to be narrowly focused upon specific applications of library work. Further, they are predominantly maintained by centralized public institutions (eg the International Federation of Library Associations, the 
Library of Congress) which are timehonored and authoritative. Conversely, there are essentially no authoritative, comprehensive or specific standards which have been authored to govern DAM as a practice. There are, of course, many metadata standards which can be applied to DAM systems, however, these hardly form a useful framework for the practitioner. Moreover, metadata standards are maintained through a variety of uncoordinated or ad hoc public and private sector entities predominantly technical in nature many of which are not ultimately authoritative. The result has been a proliferation of data description standards roughly polarized along the continuum of applications and users. The spectrum of available standards includes, on one side those that are essentially proprietary, highly specialized, and/or inextricably bound to specific information management functions and technologies: the SMPTE video metadata or the LC Encoded Archival Description standards are both pertinent examples. On the opposite side there is a much smaller group of standards which are too encyclopedic or generic for the DAM environment without significant local modifications. The Dublin Core is a member of this latter group.

The natural consequence of this decentralized environment is as one would expect - a lack of documentary standardization at both the industry and implementation levels. On every occasion when I have given a presentation on DAM, the question most raised is "Which metadata standard should we use?" Unfortunately there is no right answer, although there are many wrong answers to this enquiry, many of which I have personally discovered over the history of the MS Studios DAM implementation. Therefore, foremost of note when designing a metadata schema is that the majority of metadata standards are fairly new to the world and as such tend to deal with either the most specific or the lowest common denominator of data elements related to the content for which they were designed to describe. All standards make provisions for recording the most common properties of an asset, for example, title or description, attribution, date of production or execution, principals involved in production, talent, content abstract, type and format of the finished content, version history, etc. Positing that a DAM system is a sea-going vessel, this class of metadata elements would be the visible portion of an iceberg around which the ship must navigate, as shown in Figure 1. But upon closer examination, it becomes apparent that at least two additional classes of valuable data elements lie below the waterline, and most likely beyond the capabilities of the average metadata standard to collect.

Thus the most enigmatic of potential metadata elements are those that do not deal specifically with content identification and description, but with administrative, logistical and usage issues. While in most DAM applications a cross-section of representative data elements are hard-coded into the asset record structure, there are few, if any, that natively support the full range of administrative elements that are ultimately necessary to provide effective DAM services. Examples of administrative data elements to consider while designing a local schema include: 


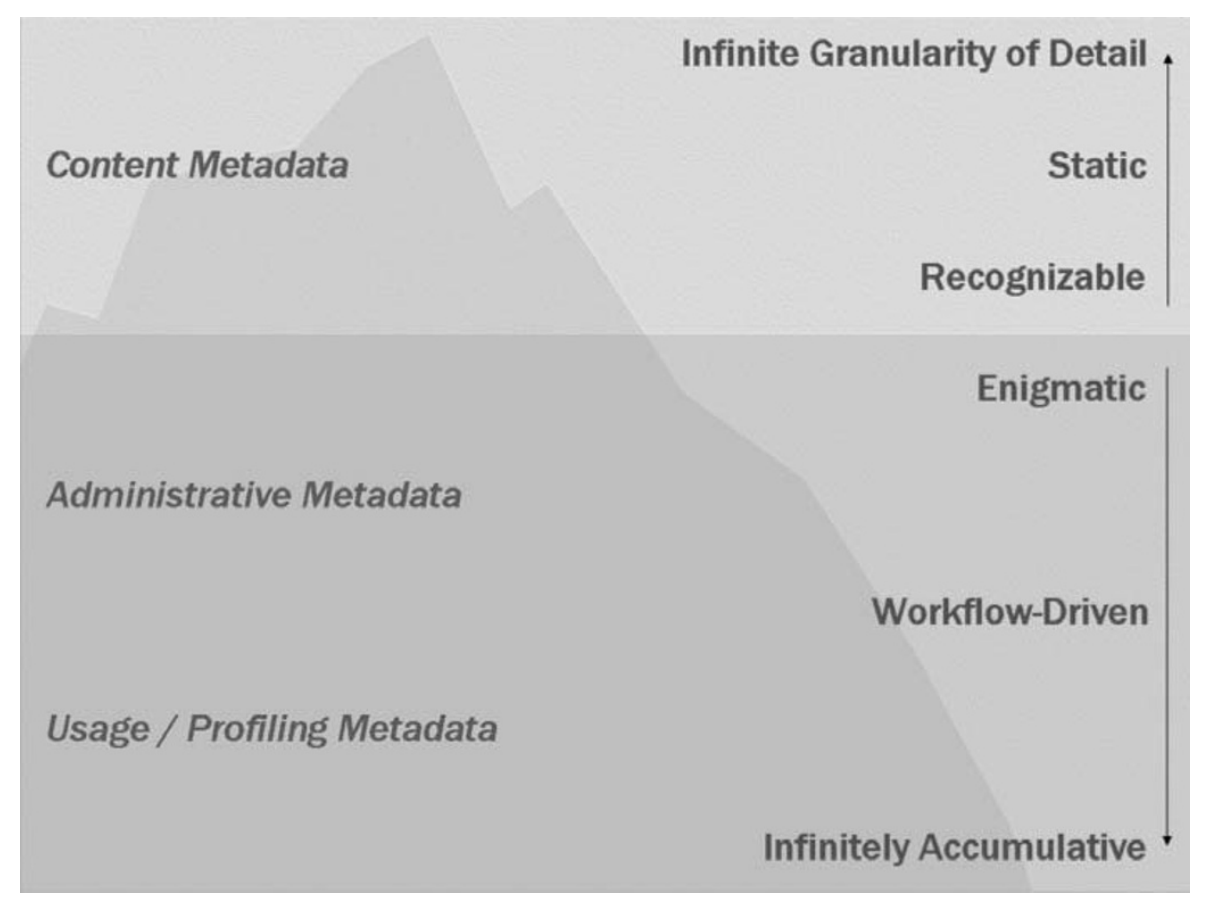

Figure 1: The metadata iceberg

details of how the content was produced; legal, contractual and access restrictions; related formats and versions; manner of use in the work environment; retention information; methods of distribution to end users; and of course, records of the larger projects or products in which it has historically been incorporated. Over time, these auxiliary elements become the most reciprocally valuable of a schema, in that they define the ongoing utility of an asset as compared with its counterparts in the repository.

Schema design is an extensive topic and beyond the scope that can be offered here. But in brief, a DAM metadata schema must not only provide an accurate conceptual representation of the content assets stored within the repository, but also of the workflow, retrieval and publication processes which are enacted upon it. The three functional stages of schema design include (1) development of an organic data model, ie a data dictionary designed for adaptability and modification based on as yet unforeseen future needs. This model should be based upon thorough documentation of all work- and dataflow interactions that the DAM system is intended to support; (2) research and evaluation of mainstream metadata standards to identify one or more that provide the best overall coverage for anticipated data management needs; and (3) harmonization of the organic and selected standardized metadata models insofar as possible, and in conformance with the DAM system's application framework. The result generally produces a hybridized schema that integrates several standards with designated local data elements. This practice is highly acceptable so long as 
the integration process is thoroughly documented. Of course, every DAM implementation project discovers the need to track local data elements that both tax the application design and for which no given standard applies. These situations must be evaluated on a caseby-case basis, and the cost-benefit analyzed to determine whether a generic tracking method is sufficient (eg recording this data in a "Notes" or similar catch-all field), or better warranted is a consultation with the manufacturer's professional services group.

\section{METADATA CAPTURE AND WORKFLOW}

In any media production environment, the introduction of DAM invariably results in the modification, or at least reconsideration of institutionalized workflow systems. Some of the customary DAM processes that impact production workflow include asset ingest, metadata capture, content search and retrieval, and project archiving. However, numerous other production factors outside the sphere of DAM also influence the means and extent by which workflow redesign is practicable. One such gating factor at MS Studios is the general practice of shooting production content on standard BetaCam videotape. After post-production processing, the final product is then encoded for live or on-demand playback, but usually also laid back onto consumer grade videotape for distribution to various audiences. This methodology requires processes for managing both videotape and digital assets, as well as capturing metadata that is applicable to both. While something less than urbane in practice, metadata were originally collected only at the end of production, when the videotapes were submitted to be archived.

The subsequent introduction of DAM at MS Studios, however, added another dimension of complexity to this process - particularly with regard to metadata collection. As shown in Figure 2, at the advent of DAM, redundant content metadata was being generated three times over the course of a production. First, after online editing, the producer would provide an initial "scratch" identification label for the edited videotapes. Secondly, the media content management staff would key this information into the video collection database (aptly named "MSMedia") when the tapes were submitted to be archived. Thirdly, the video metadata would be re-keyed into the DAM system after final video compression was completed, and the digital assets were available to be archived.

Without belaboring the inefficiency of these disconnected processes, suffice it to say that a unifying solution was designed with all possible speed. Using Microsoft Windows SharePoint Services, Microsoft Access and Visual Basic for Applications, the MS Studios Label Request Tool - despite its rather lowtech name - addressed the inefficiencies in metadata collection for both the DAM and videotape catalog systems. The application takes advantage of the MS Studios shoot tape-to-edit-tocompression production methodology, ensuring that both digital and videotape metadata are collected early on in the production process. Producers use a SharePoint request form, shown in Figure 3, to enter the requisite metadata for a production videotape - if so desired, the form can even be submitted 


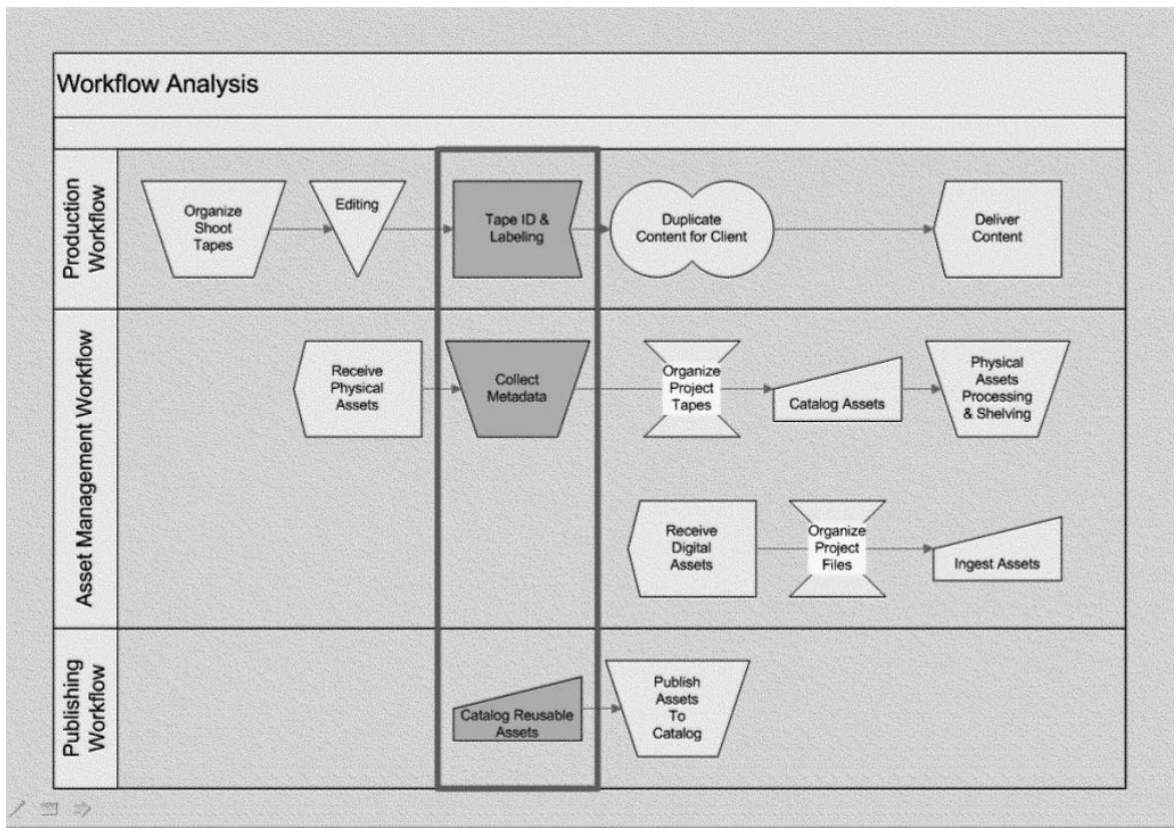

Figure 2: Original redundant metadata capture points

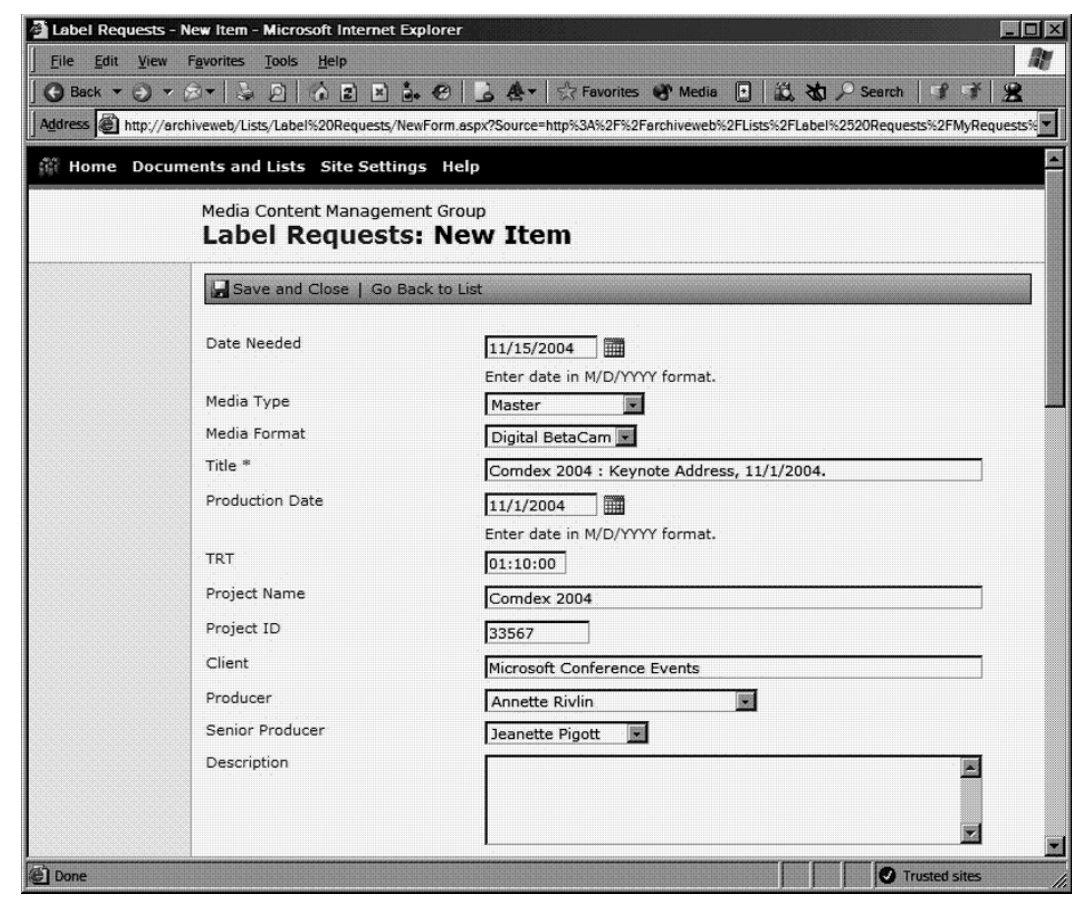

Figure 3: Producer's label request form

from inside an edit suite - and in return they are provided with standard laserprinted tape identification labels. The request form does not merely ask for identifying information, however; a wide variety of questions are asked about content access restrictions, availability for reuse, client and audience 
data, and what digital assets are expected to be generated from the tape.

Once submitted, request information is stored in a SharePoint List which is subsequently reviewed by media content management team staff through a
Microsoft Access client application called the Label Request Manager, shown in Figure 4, which allows the request to be edited and labels to be automatically printed for the requestor. Most importantly, it provides facilities for

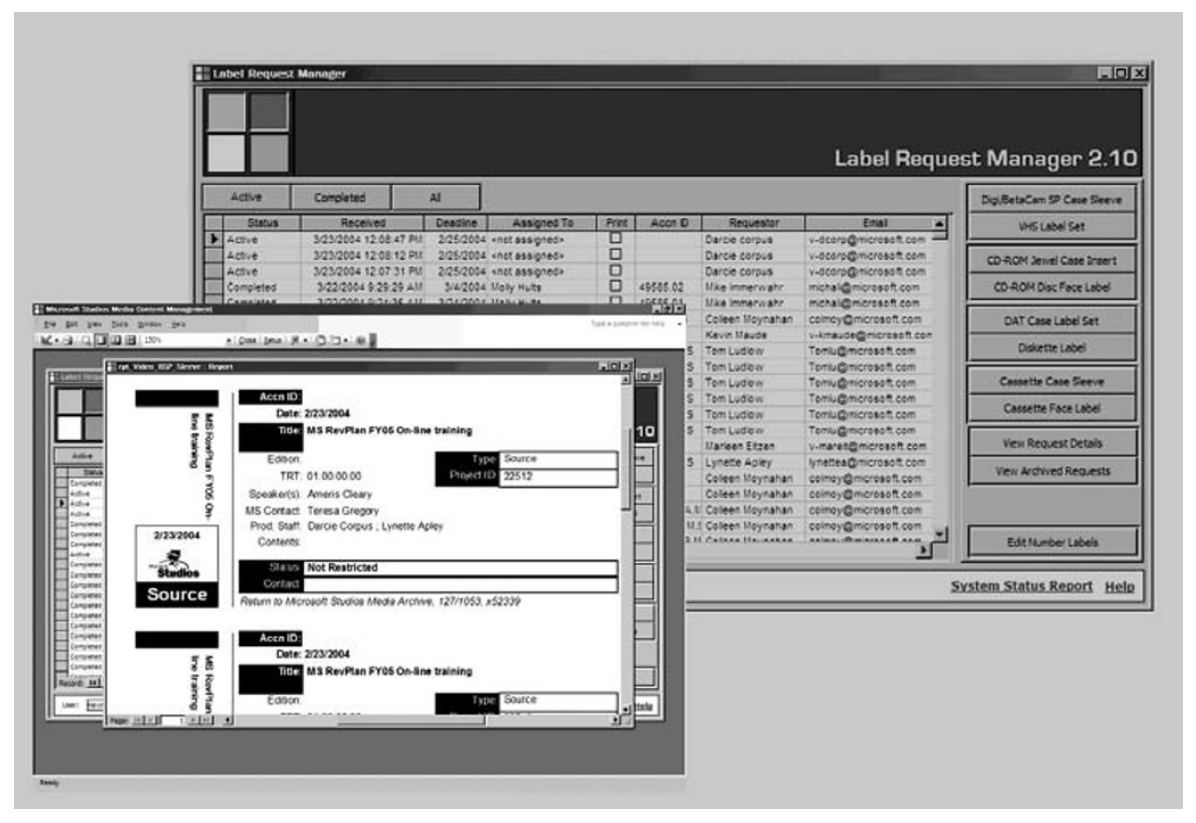

Figure 4: Label request manager

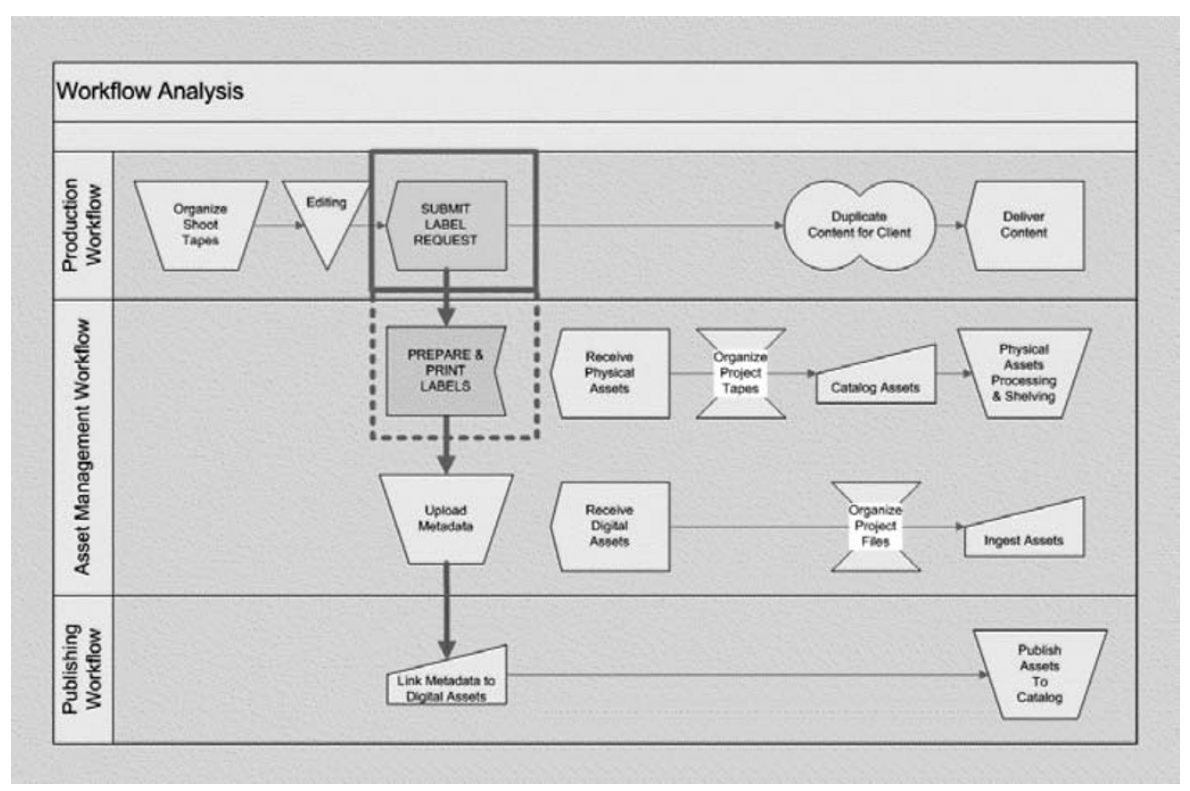

Figure 5: Streamlined metadata capture 
saving and uploading the request data directly into the videotape catalog and DAM systems, significantly reducing the data entry workload and eliminating the need to redundantly re-key asset metadata records.

As a result of this solution, the metadata capture workflow has been streamlined, transforming three redundant data entry processes into one primary data entry point, accompanied by an edit and review process, as shown in Figure 5. And because asset management metadata for both the videotape catalog and DAM system is now acquired toward the beginning, rather than at the end of production, additional efficiency is gained in that the media content management team is no longer simply reacting to the influx of new content, but can perform preparatory work over the course of a production, prior to receiving the actual content.

While a number of tools have been implemented to fully or partially automate the most laborious archival processes at MS Studios, the label request application is unique in that it engages all phases of asset management with production workflow as well as providing the production staff a reciprocally time-saving service. As such, it provides MS Studios with the first step towards integrated asset management and production workflow. 